\title{
Does coffee drinking have beneficial effects on bone health of Taiwanese adults? A longitudinal study
}

\author{
Huan-Cheng Chang ${ }^{1,2}$, Chuan-Fa Hsieh ${ }^{3,4}$, Yi-Chin Lin ${ }^{5}$, Disline Manli Tantoh ${ }^{6}$, Pei-Chieh Ko ${ }^{6}$, Ya-Yu Kung ${ }^{7}$, \\ Mei-Chi Wang ${ }^{7}$, Shu-Yi Hsu', Yi-Ching Liaw ${ }^{8}$ and Yung-Po Liaw ${ }^{6,9^{*}}$
}

\begin{abstract}
Background: Results from studies investigating the association between coffee consumption and osteoporosis or bone mineral density (BMD) have been inconsistent. This longitudinal study was performed to assess the effect of coffee drinking on bone health of Taiwanese adults.

Methods: Data were retrieved from the Li-Shin (Landseed) Hospital in Taoyuan City. In 2006, 6152 participants completed a questionnaire on coffee drinking and other lifestyle factors. In 2014, 5077 of them were followed up. Nonetheless, a total of 2395 participants with incomplete data were excluded. The final analyses included 2682 participants comprising 1195 men and 1487 women (706 premenopausal and 781 postmenopausal). T-scores were derived from the osteo-sono assessment index (OSI) which is a surrogate of BMD. Coffee drinking was categorized as "no, medium, and high" based on the number of cups that were consumed per week in both 2006 and 2014.

Results: In general, medium and high coffee drinking were associated with higher T-scores. However, significant results were observed only among high drinkers $(\beta=0.158 ; P=0.0038)$. Nonetheless, the test for linear trend was significant $(P=0.0046)$. After stratification by sex, medium and high coffee drinking were associated with higher T-scores. However, significant results were prominent only among high male drinkers $(\beta=0.237 ; P=0.0067)$ and the test for trend was significant $(P=0.0161)$. Based on menopausal status, coffee drinking was associated with higher T-scores. Nevertheless, significant results were found only among premenopausal women $(\beta=0.233 ; P=0.0355$ and $\beta=0.234 ; P=0.0152$ for medium and high coffee drinking, respectively. The test for linear trend was significant $(P=0.0108)$.
\end{abstract}

Conclusion: Coffee drinking was significantly associated with higher T-scores hence, a lower risk of osteoporosis in men and premenopausal women.

Keywords: Coffee, Osteoporosis risk, Sex, Menopausal status, Taiwan

\section{Background}

Osteoporosis is a skeletal disorder characterised by reduced bone mass and deteriorated microarchitecture of bone tissue which subsequently lead to increased bone fragility and fractures [1-3]. Low bone mineral density is among the important clinical risk factors for osteoporosis and osteoporotic fractures [1].

\footnotetext{
* Correspondence: Liawyp@csmu.edu.tw

${ }^{6}$ Department of Public Health and Institute of Public Health, Chung Shan Medical University, No. 110, Sec. 1 Jianguo N. Rd, Taichung City 40201, Taiwan

${ }^{9}$ Department of Family and Community Medicine, Chung Shan Medical University, Taichung, Taiwan

Full list of author information is available at the end of the article
}

Osteoporosis is a serious public health issue. The economic and public health burden of osteoporosis and osteoporotic fractures cannot be underemphasized. In the year 2000, the global annual incidence and prevalence of osteoporotic fractures were estimated at 8.9 and 56 million, respectively [4]. Taiwan has one of the highest hip fractures with a world population-adjusted incidence of 299/100,000 [5-7]. Between 2004 and 2011, a total of 141,397 Taiwanese aged 50 years and above had hip fractures. The incidence is estimated to increase 2.7 -fold by 2035 [7]. Osteoporotic fractures are the major source of disability in many parts of the world and huge medical costs are incurred in their management. The high medical

(c) The Author(s). 2018 Open Access This article is distributed under the terms of the Creative Commons Attribution 4.0 International License (http://creativecommons.org/licenses/by/4.0/), which permits unrestricted use, distribution, and reproduction in any medium, provided you give appropriate credit to the original author(s) and the source, provide a link to the Creative Commons license, and indicate if changes were made. The Creative Commons Public Domain Dedication waiver (http://creativecommons.org/publicdomain/zero/1.0/) applies to the data made available in this article, unless otherwise stated. 
costs result from medical examinations, hospitalization, outpatient visits, treatments, and rehabilitation [8]. It is estimated that about 10-15 billion US dollars are spent annually in the management of osteoporotic fractures [8]. In Taiwan, the annual medication cost due to fractures increased 7.2-fold (from 8.1 to 58.9 million US dollars) between 1999 and 2010 [9].

Risk factors of osteoporosis are both modifiable (can be changed) and non-modifiable (cannot be changed). Some non-modifiable factors are age, genetics, sex, and menopausal status while some modifiable factors are lifestyle, BMI, and diet [10-13]. Some lifestyle and dietary factors associated with osteoporosis include coffee drinking, cigarette smoking, alcohol consumption, and exercise [10-13]. Coffee, one of the non-alcoholic beverages is substantially consumed worldwide $[14,15]$. It contains many chemicals including caffeine, polyphenols as well as diterpenes [14, 15]. Previously, coffee was consumed mostly by the rich and celebrities in Taiwan. Nevertheless, its consumption has experienced a rapid increase recently as many people now incorporate it into their daily habits. For instance, in Taiwan, the import of coffee beans increased from about 4238 tons in 1998 to about 13,872 tons in 2007 [16]. Moreover, coffee-related products worth 28.2 billion NT dollars were imported in 2008 [16].

Results from previous studies on the association between coffee consumption and BMD or the risk of osteoporosis have not been consistent [17-20]. For instance, there was no significant association between coffee drinking and BMD among 200 postmenopausal Turkish women aged 40 years and above [17]. Similar results were observed in a study comprising 1761 premenopausal Korean women with a mean age of 36 years [18]. However, high coffee drinking was significantly associated with a small decrease in bone density but not an increased osteoporotic fracture risk among 5022 Swedish women aged over 40 years [19]. On the contrary, moderate coffee consumption was significantly associated with increased BMD among 4066 postmenopausal Korean women whose mean age was 62.6 years [20]. Because of these controversies, more investigations are warranted. Therefore, this longitudinal study was performed to assess the effect of coffee drinking on bone health of Taiwanese adults.

\section{Methods}

\section{Data source and participants}

Data used in the current longitudinal study were retrieved from the Li-Shin (Landseed) Hospital, Taoyuan, Taiwan. Individuals were included in the study if they lived in the Pinzheng District and were 30 years and above by December 31, 2005. In 2005, a total of 15,688 individuals were randomly sampled and invited for the Li-Shin prospective cohort study. Sampling was done using the proportionate stratified random sampling technique. Details about this sampling technique have been previously described [21]. Individuals were stratified by age and sex and the sample size of each group was proportionate to its size in the 2005 Pinzheng District population data. A total of 6152 individuals agreed to participate in this study.

In 2006, the 6152 individuals completed a questionnaire on lifestyle habits including coffee, alcohol drinking, exercise, diet, supplements intake, as well as smoking. They also provided information on their age, sex, educational level, and personal disease history (Additional file 1). Their bone health was determined as described below. In 2014, a total of 5077 individuals were followed up. Nonetheless, 2395 individuals were excluded from the study due to incomplete (missing) data. That is, any individual whose data were missing for at least 1 variable in either the 2006 or 2014 questionnaires were automatically excluded from the final analyses. Data from 2682 participants comprising 1195 men and 1487 women (706 premenopausal and 781 postmenopausal) were used in the final analyses (Fig. 1).

Bone health was assessed by quantitative ultrasound (QUS) using Acoustic Osteo Screener, AOS-100 (Aloka, Co. LTD, Tokyo, Japan). Details about the AOS-100 QUS device are described elsewhere [22-25]. In brief, the AOS-100 generates the index of bone density which is measured as the speed of sound (SOS) and the index of bone structure which is measured as the transmission index (TI) [24]. The osteo-sono assessment index (OSI) which reflects the bone stiffness in the calcaneus is derived from the SOS and TI as OSI $=\mathrm{TI} \times \mathrm{SOS}^{2}[22-25]$. $\mathrm{T}$-scores are generated by comparing the OSI with the mean OSI of a reference population $[24,25]$. The OSI has been shown to be strongly correlated with BMD measured by dual X-ray absorptiometry (DXA) [23]. Moreover, it is highly reproducible and has a short-term coefficient of variation of $<3 \%[2,26,27]$.

The weekly frequency of coffee consumption in 2006 (start of the study) and 2014 (follow-up) was determined by asking participants how many times $(\geq 7,5-6,3-4$, 1-2 and 0) they drank coffee per week. A five-point scale $(1-5)$ was used to represent this weekly frequency. That is, $1,2,3,4$, and 5 represented $\geq 7,5-6,3-4,1-2$ and 0 time(s)/week, respectively. One-time coffee drinking was equivalent to one cup (approximately $240 \mathrm{~mL}$ ). Coffee consumption was categorised as "no, medium, and high" by comparing the five-point scale in 2006 with that in 2014. The comparison, $5 / 5$ (i.e. 5 in 2006 and 5 in 2014) was defined as "no" consumption. Moreover, $1 / 4$, $1 / 5,2 / 4,2 / 5,3 / 4,3 / 5,4 / 4,4 / 5$ or $5 / 4$ was defined as "medium" consumption while $1 / 1,1 / 2,1 / 3,2 / 1,2 / 2,2 / 3$, $3 / 1,3 / 2,3 / 3,4 / 1,4 / 2,4 / 3,5 / 1,5 / 2$ or $5 / 3$ was defined as "high" consumption. The comparison, $5 / 5$ for example, means the participant did not drink coffee in both 2006 


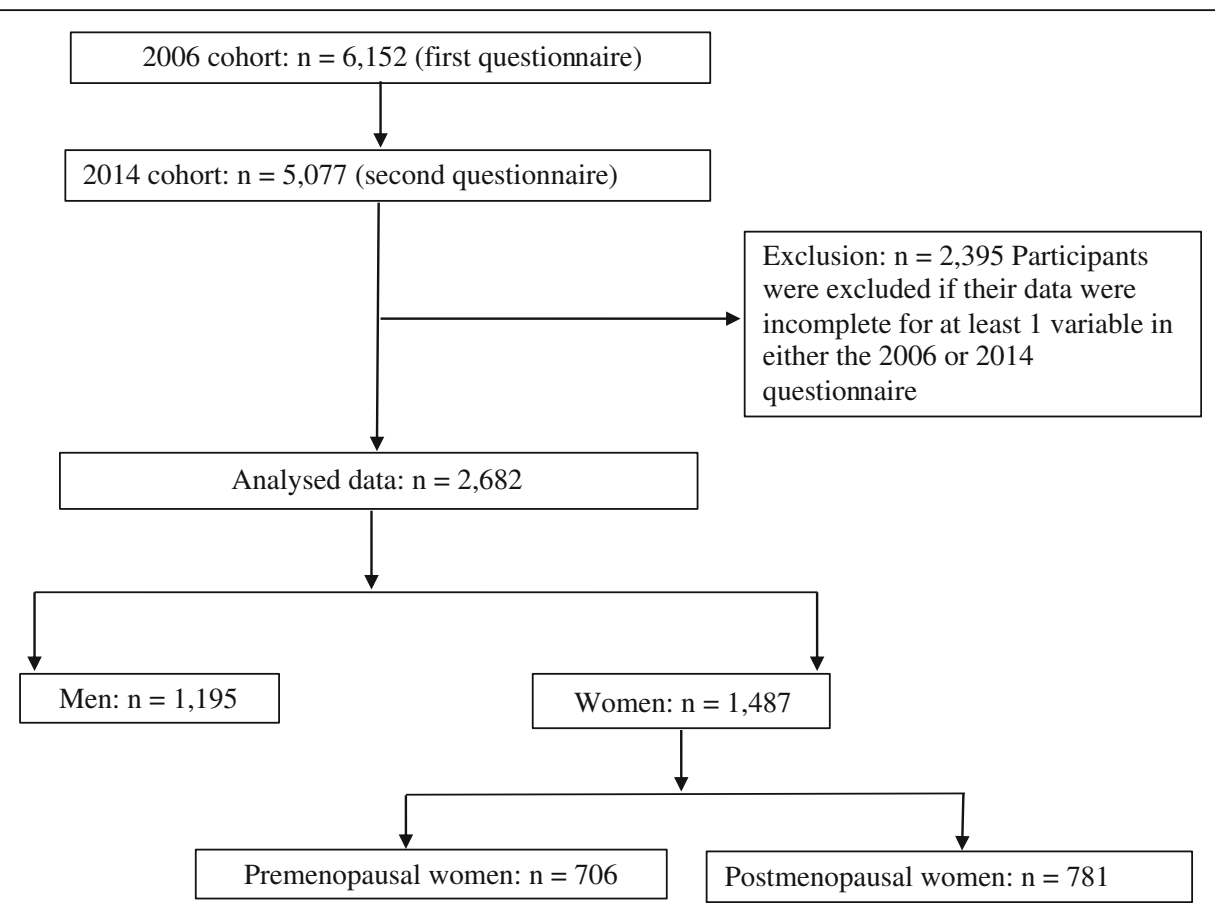

Fig. 1 Study flow chart

and 2014 while $1 / 2$ means a participant drank coffee $\geq 7$ times per week in 2006 and 5-6 times per week in 2014.

Informed consents were obtained from all participants and all methods were carried out in accordance with relevant guidelines and regulations. Ethical approval for this study was issued by the institutional review board of Li-Shin Hospital (LSHIRB No./Protocol No.16-029-B1).

\section{Statistical analyses}

The basic characteristics of the study participants were compared using the Chi-square test. The association between coffee consumption and T-scores was determined using multiple linear regression analysis. Results were presented as regression coefficients $(\beta)$ and were considered statistically significant if the $P$-value was strictly less than $0.05(P<0.05)$. Adjustments were made for confounders including age, BMI, smoking, and drinking, among others. The analyses were performed using SAS 9.4 (SAS Institute, Cary, NC, USA).

\section{Results}

Tables 1 and 2 show the basic characteristics of participants stratified by sex and menopausal status, respectively. There were significant differences between the number of male and female participants based on coffee drinking, T-scores, age, educational level, BMI, smoking, drinking, vegetarian diet, vitamin intake, and calcium intake (Table 1). Moreover, there were significant differences between the number of premenopausal and postmenopausal women based on coffee drinking, T-scores, age, educational level, BMI, exercise, diabetes, hypertension, heart disease, hyperlipidemia, and calcium intake (Table 2).

Tables 3, 4 and 5 show the association between coffee drinking and T-scores after adjustments were made for multiple confounders including age, BMI, smoking, and drinking, among others. Among all participants, medium and high coffee drinking were associated with higher T-scores, hence lower risk of osteoporosis. The regression coefficients $(\beta)$ were $0.049 ; P=0.3523$ and $0.158 ; P=0.0038$ for medium and high coffee drinking, respectively (Table 3). That is, compared to non-drinkers, T-scores among medium and high coffee drinkers were higher and the differences were 0.049 and 0.158 , respectively. Even though the results among medium drinkers were not statistically significant, a significant trend $(P=0.0046)$ was observed. That is, T-scores got higher as coffee consumption increased. In addition to coffee drinking, overweight, obesity, and exercise were significantly associated with higher T-scores $(\beta=0.181 ; P=0.0021$, $\beta=0.223 ; P=0.0049$, and $\beta=0.096 ; P=0.0305$, respectively). However, increased age, male sex, lower educational level (senior high school and below), and underweight were significantly associated with lower T-scores, hence higher osteoporosis risk. The regression coefficients $(\beta)$ were $-0.447 ; P<.0001$ for age $50-59$ years, $-0.619 ; P<.0001$ for age $\geq 70$ years, $-0.200 ; P=0.0008$ for male sex, $-0.140 ; P=0.0131$ for senior high school, $-0.257 ; P=0.0001$ for junior high school, $-0.312 ; P<.0001$ for elementary school and below, and $-0.314 ; P=0.0141$ for underweight. 
Table 1 Basic characteristics of both male and female participants

\begin{tabular}{|c|c|c|c|}
\hline Variables & $\begin{array}{l}\text { Men } \\
(n=1195)\end{array}$ & $\begin{array}{l}\text { Women } \\
(n=1487)\end{array}$ & $P$-value \\
\hline Coffee drinking & & & $0.0382^{*}$ \\
\hline No & $681(56.99)$ & $822(55.28)$ & \\
\hline Medium & $265(22.18)$ & 296(19.91) & \\
\hline High & 249(20.84) & $369(24.82)$ & \\
\hline T-score & & & $0.0004^{*}$ \\
\hline$T \geq-1$ & 477(39.92) & $679(45.66)$ & \\
\hline$-2.5<T<-1$ & 429(35.90) & 430(28.92) & \\
\hline$T \leq-2.5$ & 289(24.18) & $378(25.42)$ & \\
\hline Age group (years) & & & $<.0001^{*}$ \\
\hline $30-49$ & $471(39.41)$ & $681(45.80)$ & \\
\hline $50-69$ & $516(43.18)$ & 754(50.71) & \\
\hline$\geq 70$ & 208(17.41) & $52(3.50)$ & \\
\hline Education & & & $<.0001^{*}$ \\
\hline College and above & 367(30.71) & 243(16.34) & \\
\hline Senior high school & 403(33.72) & 475(31.94) & \\
\hline Junior high school & 182(15.23) & 278(18.70) & \\
\hline Elementary and below & 243(20.33) & 491(33.02) & \\
\hline BMI $\left(\mathrm{kg} / \mathrm{m}^{2}\right)$ & & & $<.0001^{*}$ \\
\hline Underweight & $25(2.09)$ & $51(3.43)$ & \\
\hline Normal & 462(38.66) & $756(50.84)$ & \\
\hline Overweight & $423(35.40)$ & $375(25.22)$ & \\
\hline Obese & $285(23.85)$ & $305(20.51)$ & \\
\hline Smoking & & & $<.0001^{*}$ \\
\hline Never & 597(49.96) & 1438(96.70) & \\
\hline Quit & 174(14.56) & 15(1.01) & \\
\hline Yes & $424(35.48)$ & $34(2.29)$ & \\
\hline Drinking & & & $<.0001^{*}$ \\
\hline Never & 852(71.30) & 1430(96.17) & \\
\hline Quit & $53(4.44)$ & $5(0.34)$ & \\
\hline Yes & 290(24.27) & $52(3.50)$ & \\
\hline Exercise & & & 0.6398 \\
\hline No & $421(35.23)$ & $511(34.36)$ & \\
\hline Yes & $774(64.77)$ & $976(65.64)$ & \\
\hline Vegetarian diet & & & $0.0025^{*}$ \\
\hline No & 1107(92.64) & 1327(89.24) & \\
\hline Yes & $88(7.36)$ & 160(10.76) & \\
\hline \multicolumn{4}{|l|}{ Personal disease history } \\
\hline Diabetes & $88(7.36)$ & $76(5.11)$ & $0.0155^{*}$ \\
\hline Hypertension & $231(19.33)$ & 211(14.19) & $0.0004^{*}$ \\
\hline Heart disease & $64(5.36)$ & $48(3.23)$ & $0.0062^{*}$ \\
\hline Hyperlipidemia & 84(7.03) & $80(5.38)$ & 0.0764 \\
\hline Stroke & 16(1.34) & $2(0.13)$ & $0.0001^{*}$ \\
\hline
\end{tabular}

Supplement intake
Table 1 Basic characteristics of both male and female participants (Continued)

\begin{tabular}{|c|c|c|c|}
\hline Variables & $\begin{array}{l}\text { Men } \\
(n=1195)\end{array}$ & $\begin{array}{l}\text { Women } \\
(n=1487)\end{array}$ & $P$-value \\
\hline Vitamin D & & & 0.6202 \\
\hline No & 1168(97.74) & 1449(97.44) & \\
\hline Yes & $27(2.26)$ & $38(2.56)$ & \\
\hline Other vitamins & & & $<.0001^{*}$ \\
\hline No & 1100(92.05) & 1293(86.95) & \\
\hline Yes & $95(7.95)$ & 194(13.05) & \\
\hline Calcium & & & $<.0001^{*}$ \\
\hline No & $1002(83.85)$ & 1016(68.33) & \\
\hline Yes & 193(16.15) & $471(31.67)$ & \\
\hline
\end{tabular}

When stratified by sex, coffee drinking was associated with higher T-scores in both men and women (Table 4). However, significant results were prominent only among men with medium coffee drinking $(\beta=0.237 ; P=0.0067)$. A significant trend $(P=0.0161)$ was also prominent only among men. Overweight, exercise, and vitamin intake were also significantly associated with higher T-scores among men $(\beta=0.272 ; P=0.0011, \beta=0.162 ; P=0.0172$, and $\beta=0.377 ; P=0.0167$, respectively). Nevertheless, increased age and lower educational level (junior high school and below) were significantly associated with lower T-scores among both sexes. Among men, the regression coefficients $(\beta)$ were $-0.334 ; P<.0001$ for age 50-60 years, $-0.482 ; P<.0001$ for age $\geq 70$ years, -0.245 ; $P=0.0135$ for junior high school and $-0.228 ; P=0.0246$ for elementary school and below. Among women, regression coefficients $(\beta)$ were $-0.388 ; P<.0001$ for age 50-60 years, $-0.829 ; P<.0001$ for age $\geq 70$ years, -0.245 ; $P=0.0116$ for junior high school and $-0.248 ; P=0.0124$ for elementary school and below.

Based on menopausal status, both medium and high coffee drinking were significantly associated with higher $\mathrm{T}$-scores among premenopausal women $(\beta=0.233$; $P=0.0355$ for medium and $\beta=0.234 ; P=0.0152$ for high coffee drinking). In addition, a significant trend $(P=0.0108)$ was prominent (Table 5). Increased age was significantly associated with lower $\mathrm{T}$-scores in postmenopausal women $(\beta=-0.565 ; P<.0001$ and $\beta=-0.983 ; P<.0001$ for age $50-69$ and $\geq 70$ years, respectively). However, lower educational level (senior high school and elementary school and below) was significantly associated with lower T-scores only among premenopausal women $(\beta=-0.249 ; P=0.0123$ for senior high school and $\beta=-0.388 ; P=0.0255$ for elementary school and below).

\section{Discussion}

In the current study, coffee drinking was significantly associated with higher T-scores among men and premenopausal 
Table 2 Basic characteristics of premenopausal and postmenopausal women

\begin{tabular}{|c|c|c|c|}
\hline Variables & $\begin{array}{l}\text { Premenopausal } \\
\text { women } n(\%)\end{array}$ & $\begin{array}{l}\text { Postmenopausal } \\
\text { women } n(\%)\end{array}$ & $P$-value \\
\hline Coffee drinking & & & $<.0001^{*}$ \\
\hline No & $332(47.03)$ & $490(62.74)$ & \\
\hline Medium & $146(20.68)$ & $150(19.21)$ & \\
\hline High & $228(32.29)$ & $141(18.05)$ & \\
\hline T-score & & & $<.0001^{*}$ \\
\hline$T \geq-1$ & $410(58.07)$ & 269 (34.44) & \\
\hline$-2.5<T<-1$ & 115 (16.29) & $315(40.33)$ & \\
\hline $\mathrm{T} \leq-2.5$ & $181(25.64)$ & $197(25.22)$ & \\
\hline Age group (years) & & & $<.0001^{*}$ \\
\hline $30-49$ & 607 (85.98) & $74(9.48)$ & \\
\hline $50-69$ & 99 (14.02) & $655(83.87)$ & \\
\hline$\geq 70$ & $0(0.00)$ & $52(6.66)$ & \\
\hline Education & & & $<.0001^{*}$ \\
\hline College and above & $197(27.90)$ & $46(5.89)$ & \\
\hline Senior high school & $322(45.61)$ & 153 (19.59) & \\
\hline Junior high school & 127 (17.99) & $151(19.33)$ & \\
\hline Elementary and below & $60(8.50)$ & $431(55.19)$ & \\
\hline BMI $\left(\mathrm{kg} / \mathrm{m}^{2}\right)$ & & & $<.0001^{*}$ \\
\hline Underweight & $35(4.96)$ & $16(2.05)$ & \\
\hline Normal & 419 (59.35) & 337 (43.15) & \\
\hline Overweight & $145(20.54)$ & $230(29.45)$ & \\
\hline Obese & $107(15.16)$ & $198(25.35)$ & \\
\hline Smoking & & & 0.1044 \\
\hline Never & 677 (95.89) & $761(97.44)$ & \\
\hline Quit & $11(1.56)$ & $4(0.51)$ & \\
\hline Yes & $18(2.55)$ & $16(2.05)$ & \\
\hline Drinking & & & 0.0532 \\
\hline Never & $670(94.90)$ & $760(97.31)$ & \\
\hline Quit & $3(0.42)$ & $2(0.26)$ & \\
\hline Yes & $33(4.67)$ & $19(2.43)$ & \\
\hline Exercise & & & $0.0001^{*}$ \\
\hline No & 278 (39.38) & $233(29.83)$ & \\
\hline Yes & $428(60.62)$ & $548(70.17)$ & \\
\hline Vegetarian diet & & & 0.0661 \\
\hline No & $641(90.79)$ & $686(87.84)$ & \\
\hline Yes & $65(9.21)$ & $95(12.16)$ & \\
\hline \multicolumn{4}{|l|}{ Personal disease history } \\
\hline Diabetes & $13(1.84)$ & $63(8.07)$ & $<.0001^{*}$ \\
\hline Hypertension & $45(6.37)$ & $166(21.25)$ & $<.0001^{*}$ \\
\hline Heart disease & $15(2.12)$ & $33(4.23)$ & $0.0221^{*}$ \\
\hline Hyperlipidemia & $18(2.55)$ & $62(7.94)$ & $<.0001^{*}$ \\
\hline Stroke & $1(0.14)$ & $1(0.13)$ & 0.9430 \\
\hline
\end{tabular}

Table 2 Basic characteristics of premenopausal and postmenopausal women (Continued)

\begin{tabular}{llll}
\hline Variables & $\begin{array}{l}\text { Premenopausal } \\
\text { women } n(\%)\end{array}$ & $\begin{array}{l}\text { Postmenopausal } \\
\text { women } n(\%)\end{array}$ & $\begin{array}{c}P \text {-value } \\
\text { Vitamin D }\end{array}$ \\
$\quad 688(97.45)$ & $761(97.44)$ & 0.9891 \\
No & $18(2.55)$ & $20(2.56)$ & \\
Yes & & & 0.0619 \\
$\quad$ No & $626(88.67)$ & $667(85.40)$ & \\
Yes & $80(11.33)$ & $114(14.60)$ & \\
Calcium & & & $<.0001^{*}$ \\
No & $534(75.64)$ & $482(61.72)$ & \\
Yes & $172(24.36)$ & $299(38.28)$ & \\
${ }^{*} P<0.05$ & & &
\end{tabular}

women. As far as we know, this study is among the first to demonstrate the protective effect of coffee drinking on osteoporosis risk among premenopausal women. Although osteoporosis is more common in postmenopausal women, its occurrence in men and premenopausal women cannot be ignored. Therefore increasing the bone mass of men and both premenopausal and menopausal women can serve as a preventive measure against bone loss and subsequently osteoporosis [3]. The strength of the current study is that participants were stratified by sex and menopausal status using information obtained from two questionnaires (2006 and 2014) and analysis was done using the multiple linear regression model which adjusted for many confounders.

So far, the association between coffee drinking and BMD or the risk of osteoporosis has been incoherent. In line with the current study, moderate coffee consumption was significantly associated with increased BMD among 992 Chinese men with a mean age of 64.11 years [3]. Moreover, coffee consumption was associated with increased broadband ultrasound attenuation (BUA), hence a lower risk of osteoporosis among 344 Malaysian women aged 50 years and above [28]. Besides, coffee drinking at the premenopausal stage was not significantly associated with BMD among 200 postmenopausal Turkish women aged 40 years [17]. Furthermore, in a cross-sectional study involving 1336 premenopausal and 1593 postmenopausal Taiwanese aged 30 years and above, coffee consumption was significantly associated with decreased risk of osteoporosis in premenopausal, but not postmenopausal women [29]. Unlike the current study, coffee drinking was not significantly associated BMD among 1761 premenopausal Korean women with a mean age of 36 years [18]. Moreover, it was significantly associated with decreased BMD among 258 healthy Polish men aged 40-63 years [30]. In addition, it was significantly associated with decreased BMD among men in a Swedish cohort comprising 359 men and 358 women aged 70 years [31]. It was also associated with 
Table 3 Multiple linear regression analysis showing the association between coffee drinking and T-scores among study participants

\begin{tabular}{lll}
\hline Variables & $\beta$ & $P$-value \\
\hline Coffee drinking (Reference: No) & - & \\
Medium & 0.049 & 0.3523 \\
High & 0.158 & $0.0038^{*}$ \\
P-trend & $0.0046^{*}$ &
\end{tabular}

Age group (Reference: $30-49$ years)

$\begin{array}{lll}50-69 & -0.447 & <.0001^{*} \\ \geq 70 & -0.619 & <.0001^{*}\end{array}$

Sex (Reference: Women)

Men

Education (Reference:=College and above)

$$
\begin{aligned}
& \text { Senior high school } \\
& \text { Junior high school }
\end{aligned}
$$

Elementary and below

BMI (Reference: Normal)

$$
\begin{aligned}
& \text { Underweight } \\
& \text { Overweight }
\end{aligned}
$$

Obese

$$
\begin{aligned}
& -0.140 \\
& -0.257 \\
& -0.312
\end{aligned}
$$

0.223

Smoking (Reference: Never)

$$
\text { Quit }
$$

Yes

Drinking (Reference: Never)

$$
\text { Quit }
$$$$
\text { Yes }
$$$$
-0.094
$$

.8427

0.1546

Quit

Exercise (Reference: No)

Yes

Vegetarian diet (Reference: No)

Yes

$$
-0.038
$$

0.7940

0.8607

Personal disease history

$$
\begin{aligned}
& \text { Diabetes } \\
& \text { Hypertension } \\
& \text { Heart disease } \\
& \text { Hyperlipidemia } \\
& \text { Stroke }
\end{aligned}
$$

\begin{tabular}{|c|c|c|c|c|}
\hline \multirow[t]{2}{*}{ Variables } & \multicolumn{2}{|l|}{ Men } & \multicolumn{2}{|c|}{ Women } \\
\hline & $\beta$ & $P$-value & $\beta$ & $P$-value \\
\hline \multicolumn{5}{|c|}{ Coffee drinking (Reference: No) } \\
\hline Medium & 0.003 & 0.9662 & 0.105 & 0.1445 \\
\hline High & 0.237 & $0.0067^{*}$ & 0.101 & 0.1492 \\
\hline P-trend & \multicolumn{2}{|c|}{$0.0161^{*}$} & \multicolumn{2}{|c|}{0.0947} \\
\hline
\end{tabular}

$-0.016$

Supplement intake

$$
\begin{aligned}
& \text { Vitamin D (Reference: No) } \\
& \text { Yes }
\end{aligned}
$$$$
0.006
$$

Other vitamins (Reference: No)

Yes

Calcium (Reference: No)

$$
\text { Yes }
$$

Table 4 Multiple linear regression analysis showing the association between coffee drinking and T-scores among men and women

Age group (Reference: $30-49$ years)

$$
\begin{array}{lllll}
50-69 & -0.334 & <.0001^{*} & -0.388 & <.0001^{*} \\
\geq 70 & -0.482 & <.0001^{*} & -0.829 & <.0001^{*}
\end{array}
$$

Education (Reference: College and above)

$\begin{array}{lllll}\text { Senior high school } & -0.085 & 0.2740 & -0.169 & 0.0458 \\ \text { Junior high school } & -0.245 & 0.0135^{*} & -0.245 & 0.0116^{*} \\ \text { Elementary and below } & -0.228 & 0.0246^{*} & -0.248 & 0.0124^{*} \\ \text { BMI (Reference: Normal) } & & & & \\ \text { Underweight } & -0.369 & 0.0775 & -0.258 & 0.1133 \\ \text { Overweight } & 0.272 & 0.0011^{*} & 0.082 & 0.3383 \\ \text { Obese } & 0.325 & 0.0049 & 0.111 & 0.3172 \\ \text { Smoking (Reference: Never) } & & & & \\ \text { Quit } & -0.086 & 0.3410 & 0.340 & 0.3238 \\ \text { Yes } & -0.128 & 0.0800 & 0.211 & 0.2604 \\ \text { Drinking (Reference: Never) } & & & & \\ \text { Quit } & -0.020 & 0.8973 & 0.190 & 0.7787 \\ \text { Yes } & 0.011 & 0.8865 & -0.011 & 0.9495\end{array}$

Exercise (Reference: No)

$\begin{array}{lllll}\text { Yes } & 0.162 & 0.0172^{*} & 0.037 & 0.5339\end{array}$

Vegetarian diet (Reference: No)

$\begin{array}{lllll}\text { Yes } & -0.059 & 0.4502 & 0.025 & 0.4599\end{array}$

Personal disease history

$\begin{array}{lllll}\text { Diabetes } & -0.236 & 0.1169 & -0.129 & 0.4031 \\ \text { Hypertension } & 0.004 & 0.9657 & 0.049 & 0.6030 \\ \text { Heart disease } & 0.118 & 0.4049 & -0.065 & 0.6822 \\ \text { Hyperlipidemia } & -0.013 & 0.9183 & 0.084 & 0.5012 \\ \text { Stroke } & 0.029 & 0.9162 & 1.564 & 0.0177^{*}\end{array}$

Supplement intake

Vitamin D (Reference: No)

$\begin{array}{lllll}\text { Yes } & 0.053 & 0.8028 & -0.049 & 0.7669\end{array}$

Other vitamins (Reference: No)

$\begin{array}{lllll}\text { Yes } & 0.377 & 0.0167^{*} & -0.022 & 0.8250\end{array}$

Calcium (Reference: No)

$\begin{array}{lllll}\text { Yes } & -0.272 & 0.0159^{*} & -0.053 & 0.4692\end{array}$

${ }^{*} P<0.05$. Adjusted for age, education, BMl, smoking, drinking, exercise, vegetarian diet, vitamin $D$, other vitamins, calcium, blood type, fasting blood glucose, creatinine, uric acid, total cholesterol, triglycerides, HDL, LDL, GOT, GPT, SBP, DBP, waist circumference, waist-hip ratio, body fat, diabetes, hypertension, heart disease, hyperlipidemia and stroke

0.05. Adjusted for age, education, BMl, smoking, drinking, exercise, vegetarian diet, vitamin $\mathrm{D}$, other vitamins, calcium, blood type, fasting blood glucose, creatinine, uric acid, total cholesterol, triglycerides, HDL, LDL, GOT, GPT, SBP, DBP, waist circumference, waist-hip ratio, body fat, diabetes, hypertension, heart disease, hyperlipidemia and stroke 
Table 5 Multiple linear regression analysis showing the association between coffee drinking and T-scores among premenopausal and postmenopausal women

\begin{tabular}{|c|c|c|c|c|}
\hline \multirow[t]{2}{*}{ Variables } & \multicolumn{2}{|c|}{$\begin{array}{l}\text { Premenopausal } \\
\text { women }\end{array}$} & \multicolumn{2}{|c|}{$\begin{array}{l}\text { Postmenopausal } \\
\text { women }\end{array}$} \\
\hline & $\beta$ & $P$-value & $\beta$ & $P$-value \\
\hline \multicolumn{5}{|l|}{ Coffee drinking (Ref: No) } \\
\hline Medium & 0.233 & $0.0355^{*}$ & 0.037 & 0.7061 \\
\hline High & 0.234 & $0.0152^{*}$ & -0.088 & 0.4108 \\
\hline P-trend & $0.0108^{*}$ & & 0.5399 & \\
\hline \multicolumn{5}{|c|}{ Age group (Ref: $30-49$ years) } \\
\hline $50-69$ & -0.218 & 0.0881 & -0.565 & $<.0001^{*}$ \\
\hline$\geq 70$ & - & - & -0.983 & $<.0001^{*}$ \\
\hline \multicolumn{5}{|c|}{ Educational (Ref: College and above) } \\
\hline Senior high school & -0.249 & $0.0123^{*}$ & -0.010 & 0.9563 \\
\hline Junior high school & -0.255 & 0.0510 & -0.195 & 0.2658 \\
\hline Elementary and below & -0.388 & $0.0255^{*}$ & -0.123 & 0.4573 \\
\hline \multicolumn{5}{|l|}{ BMI (Ref: Normal) } \\
\hline Underweight & -0.134 & 0.5282 & -0.430 & 0.1036 \\
\hline Overweight & 0.209 & 0.1216 & 0.033 & 0.7723 \\
\hline Obese & 0.218 & 0.2334 & 0.052 & 0.7139 \\
\hline \multicolumn{5}{|l|}{ Smoking (Ref: Never) } \\
\hline Quit & 0.668 & 0.0808 & -0.801 & 0.4042 \\
\hline Yes & 0.465 & 0.0605 & -0.148 & 0.6212 \\
\hline \multicolumn{5}{|l|}{ Drinking (Ref: Never) } \\
\hline Quit & 0.096 & 0.8942 & - & - \\
\hline Yes & -0.025 & 0.9083 & -0.048 & 0.8694 \\
\hline \multicolumn{5}{|l|}{ Exercise (Ref: No) } \\
\hline Yes & -0.028 & 0.7542 & 0.090 & 0.2737 \\
\hline \multicolumn{5}{|l|}{ Vegetarian diet (Ref: No) } \\
\hline Yes & 0.024 & 0.7706 & 0.019 & 0.6070 \\
\hline \multicolumn{5}{|l|}{ Personal disease history } \\
\hline Diabetes & -0.306 & 0.3908 & -0.244 & 0.1732 \\
\hline Hypertension & 0.327 & 0.1440 & -0.052 & 0.6207 \\
\hline Heart disease & 0.241 & 0.3829 & -0.187 & 0.3447 \\
\hline Hyperlipidemia & -0.295 & 0.2992 & 0.206 & 0.1420 \\
\hline Stroke & 0.200 & 0.8335 & 2.624 & $0.0055^{*}$ \\
\hline \multicolumn{5}{|l|}{ Supplements } \\
\hline \multicolumn{5}{|l|}{ Vitamin D (Ref: No) } \\
\hline Yes & -0.242 & 0.3356 & 0.045 & 0.8370 \\
\hline \multicolumn{5}{|l|}{ Other vitamins (Ref: No) } \\
\hline Yes & -0.116 & 0.5060 & 0.046 & 0.7087 \\
\hline \multicolumn{5}{|l|}{ Calcium (Ref: No) } \\
\hline Yes & 0.039 & 0.7670 & -0.088 & 0.3244 \\
\hline
\end{tabular}

${ }^{*} P<0.05$. Adjusted for age, education, BMl, smoking, drinking, exercise, vegetarian diet, vitamin $D$, other vitamins, calcium, blood type, fasting blood glucose, creatinine, uric acid, total cholesterol, triglycerides, HDL, LDL, GOT, GPT, SBP, DBP, waist circumference, waist-hip ratio, body fat, diabetes, hypertension, heart disease, hyperlipidemia and stroke lower BMD among 100 postmenopausal women aged 50-65 years who lived in Sarajevo [32]. Furthermore, high coffee drinking was significantly associated with a small decrease in bone mineral density but not an increased osteoporotic fracture risk among 5022 Swedish women aged over 40 years [19]. Moderate coffee consumption was significantly associated with increased BMD among 4066 postmenopausal Korean women with a mean age of 62.6 years [20]. Moreover, coffee consumption was significantly associated with increased BMD among 1817 Chinese postmenopausal women [33].

The mechanism behind the beneficial effects of coffee on bone health is still unclear. However, it can be partly explained in terms of its antioxidant and anti-inflammatory properties. For instance, coffee is composed of high polyphenols (chlorogenic acids) which have the potential to inhibit osteoclastogenesis hence, reduction of osteoporosis risk [3, 20, 34].

The use of QUS might be one of the factors contributing to the discrepancies between this study and previous ones. Moreover, these discrepancies can be accounted for in terms of differences in sample sizes, ethnicities, study designs, statistical methods, ages, sex, as well as confounders. For instance, in this study, coffee drinking was associated with higher T-scores. The study was longitudinal in nature and participants included both Taiwanese men $(n=1195)$ and women $(n=1487)$ aged 30 years and above. The female participants were further stratified by menopausal status. Moreover, linear regression was used to determine the association between T-scores and coffee drinking and adjustments were made for important confounders like age, BMI, smoking, exercise, and diet, among others. However, in a study conducted by Choi and colleagues, there was no significant association between coffee drinking and BMD. The study was cross-sectional in nature and participants comprised only Korean premenopausal women $(n=1761)$ with a mean age of 36 [18]. In another previous study, coffee consumption was significantly associated with decreased BMD. Participants consisted of 258 Polish men aged 40-63 years. The relationship between coffee and BMD was determined by analysis of variance (ANOVA) and no adjustments were made for confounders [30]. Furthermore, in another study where both men $(n=359)$ and women $(n=358)$ aged 70 years were included, coffee drinking was significantly associated with decreased BMD. Moreover, the effect of cytochrome P450 1A2 (CYP1A2) genotype was considered in the study [31].

The frequency of coffee consumption is important when determining the relationship between coffee consumption and bone health. For instance, compared to 1 cup per day, the consumption of 4 or more cups of coffee per day was found to be significantly associated with increased osteoporotic fracture risk among 31,527 
Swedish women aged 40-76 years with low calcium consumption [35]. Moreover, the consumption of 4 or more cups of coffee per day led to a reduction in male femoral BMD compared with lower volumes. However, there was no such observation in women [31]. In a cohort of Swedish women aged above 40 years, consuming 4 or more cups of coffee per day led to decreased bone density. However, this did not increase the osteoporotic fracture risk [19]. Similarly, in a cohort of Swedish men, consuming 4 or more cups of coffee per day was not associated with osteoporotic fracture risk [36]. In the current study, the frequency of coffee consumption was also considered. The questionnaires used contained information on how many times $(\geq 7,5-6,3-4,1-2$ and 0$)$ participants consumed coffee in a week and a five-point scale was used to represent this weekly frequency as stated in the methods section. The grouping of coffee drinking into "no, medium and high" was based on a combination of the weekly frequency of consumption in both 2006 and 2014 questionnaires.

In line with this study, exercise has been previous demonstrated to be associated with higher BMD [3740]. It would be better to have assessed the relation between weight-bearing exercise and calcaneal OSI in detail. However, this study did not focus on the exercise type. Further investigation taking the exercise type into consideration is recommended. Increased BMD was also associated with obesity and overweight $[28,40,41]$. The association of obesity/overweight with BMD is probably due to the conversion of androgen to estrogen which increases bone mass [40, 42]. Another plausible explanation is the higher mechanical loading in obese and overweight individuals (higher BMI) [28, 42]. Low BMI [41, 43, 44], age [3, 6, $39,43,44]$ and education below college level $[39,44,45]$ have been associated with lower BMD. Low bone mass is associated with increased age because as time progresses, the bone tissue's synthesizing capacity reduces [44].

The limitation of this study is that bone health was assessed by QUS. Unlike DXA, bone health assessment using QUS devices have a lot of variations as far as precision, T-score, reference ranges are concerned $[2,46]$. Therefore, in clinical practice, QUS is not a gold standard for BMD measurement $[2,46]$. It only serves as a screening guide for patients to go for a more definitive test like DXA. However, OSI measured by QUS has been shown to be closely correlated $(r=0.87)$ with BMD measured by DXA [23]. Furthermore, QUS devices are non-ionizing (safe), portable and cost-effective. Nonetheless, the use of QUS devices in assessing the risk of fracture is very pertinent in developing countries especially in the absence of DXA.

In conclusion, coffee drinking was significantly associated with higher T-scores, hence a lower risk of osteoporosis in men and premenopausal women. Future studies using DXA are recommended to confirm these findings.

\section{Additional file}

\section{Additional file 1: Translated questionnaire. (PDF 1664 kb)}

\section{Abbreviations}

ANOVA: Analysis of variance analysis; AOS: Acoustic Osteo-screener; BMD: Bone mineral density; BMl: Body mass index; BUA: Broadband ultrasound attenuation; CYP1A2: Cytochrome P450 1A2; DBP: Diastolic blood pressure; DXA: Dual-energy X-ray absorptiometry; GOT: Glutamic-oxaloacetic transaminase; GPT: Glutamic-pyruvic transaminase; HDL: High-density lipoprotein; LDL: Low-density lipoprotein; QUS: Quantitative ultrasound; SBP: Systolic blood pressure

\section{Acknowledgements}

Authors are grateful to the following persons for assisting in translating the questionnaire: Ji-Han Zhong, Oswald Ndi Nfor, Wen-Yu Lu, Pei-Hsin Chen, Kuan-Jung Lee, Nokuphila Balindile Ndzinisa, Chih-Hsuan Hsiao, Chi-Che Hong and Wen- Hsiu Liu.

\section{Funding}

This work was partly supported by the Ministry of Science and Technology (MOST), Taiwan (MOST 107-2627-M-040-002 and funds from the collaborative projects between Chung Shan Medical University and Li-Shin (Landseed) Hospital (CSMU-LSH-103-02, CSMU-LSH-104-01, CSMU-LSH-105-01, and CSMULSH-106-01) awarded to Prof. Yung-Po Liaw. Data used in this study were retrieved from the Li-Shin (Landseed) Hospital.

\section{Availability of data and materials}

The data that support the findings of this study are available from the Li-Shin (Landseed) Hospital but restrictions apply to the availability of these data, which were used under license for the current study, and so are not publicly available. Data are however available from the authors upon reasonable request and with permission of the Li-Shin (Landseed) Hospital.

\section{Authors' contributions}

$\mathrm{H}-\mathrm{CC}, \mathrm{C}-\mathrm{FH}, \mathrm{Y}-\mathrm{YK}, \mathrm{M}-\mathrm{C} \mathrm{W}, \mathrm{Y}-\mathrm{CL}, \mathrm{S}-\mathrm{YH}$, and $\mathrm{Y}-\mathrm{PL}$ conceived the experiments. $\mathrm{P}-\mathrm{CK}$ and $\mathrm{Y}-\mathrm{PL}$ were involved in data management and statistical analysis. H-CC, DMT, Y-YK, M-C W, Y-CL, and Y-PL were responsible for the data interpretation and drafting of the manuscript. $Y-P L$ and DMT critically reviewed the manuscript for intellectual contents. All authors read and approved the final manuscript.

Ethics approval and consent to participate

Participants signed informed consents prior to data collection. The study was approved by the institutional review board of Li-Shin (Landseed) Hospital (LSHIRB No./Protocol No.: 16-029-B1).

Consent for publication

Not applicable.

\section{Competing interests}

The authors declare that they have no competing interests.

\section{Publisher's Note}

Springer Nature remains neutral with regard to jurisdictional claims in published maps and institutional affiliations.

\section{Author details}

${ }^{1}$ Division of Family Medicine, Department of Community Medicine, Landseed Hospital, Taoyuan, Taiwan. Department of Health Care Management, Chang Gung University, Taoyuan, Taiwan. ${ }^{3}$ Department of Medical Education and Research, Landseed Hospital, Taoyuan, Taiwan. ${ }^{4}$ Center for General Education, Hsin Sheng College of Medical Care and Management, Taoyuan, Taiwan. ${ }^{5}$ Institute of Nutritional Science, Chung Shan Medical University, Taichung, Taiwan. ${ }^{6}$ Department of Public Health and Institute of Public Health, Chung Shan Medical University, No. 110, Sec. 1 Jianguo N. Rd, Taichung City 40201, Taiwan. ${ }^{7}$ Division of Health Management, Landseed Hospital, Taoyuan,

Taiwan. ${ }^{8}$ Institute of Clinical Medicine, National Yang-Ming University, Taipei, 
Taiwan. ${ }^{9}$ Department of Family and Community Medicine, Chung Shan Medical University, Taichung, Taiwan.

\section{Received: 13 March 2018 Accepted: 30 October 2018 Published online: 20 November 2018}

\section{References}

1. Kanis J, McCloskey E, Johansson H, Cooper C, Rizzoli R, Reginster J-Y. European guidance for the diagnosis and management of osteoporosis in postmenopausal women. Osteoporosis Int. 2013;24(1):23-57.

2. Krieg M-A, Barkmann R, Gonnelli S, Stewart A, Bauer DC, Barquero LDR, Kaufman JJ, Lorenc R, Miller PD, Olszynski WP. Quantitative ultrasound in the management of osteoporosis: the 2007 ISCD official positions. J Clin Densitom. 2008;11(1):163-87.

3. Yu Q, Liu Z-H, Lei T, Tang Z. Subjective evaluation of the frequency of coffee intake and relationship to osteoporosis in Chinese men. J Health Popul Nutr. 2016;35(1):24.

4. Johnell $\mathrm{O}$, Kanis J. An estimate of the worldwide prevalence and disability associated with osteoporotic fractures. Osteoporosis Int. 2006;17(12):1726-33.

5. Kanis JA, Odén A, McCloskey E, Johansson H, Wahl DA, Cooper C. A systematic review of hip fracture incidence and probability of fracture worldwide. Osteoporosis Int. 2012;23(9):2239-56.

6. Lu Y-C, Lin YC, Lin Y-K, Liu Y-J, Chang K-H, Chieng P-U, Chan WP. Prevalence of osteoporosis and low bone mass in older chinese population based on bone mineral density at multiple skeletal sites. Sci Rep. 2016;6.

7. Chen $\mathrm{H}$, Chiang C-Y, Li Y-H, Chang C-H, Hu C-C, Chen D, Chang Y, Yang W-E, Shih H-N, Ueng S-N. Nationwide cohort study of hip fractures: time trends in the incidence rates and projections up to 2035. Osteoporosis Int. 2015;26(2):681-8.

8. Khodadadi S, Khodadadi S. Estimation of annual per capita treatment in osteoporosis. JPD. 2016:4(1):18

9. Chan D-C, Lee Y-S, Wu Y-J, Tsou H-H, Chen C-T, Hwang J-S, Tsai K-S, Yang R-S. A 12-year ecological study of hip fracture rates among older Taiwanese adults. Calcif Tissue Int. 2013:93(5):397-404.

10. Silva TR, Franz R, Maturana MA, Spritzer PM. Associations between body composition and lifestyle factors with bone mineral density according to time since menopause in women from southern Brazil: a cross-sectional study. BMC Endocr Disord. 2015;15(1):71.

11. Zhu K, Hunter M, James A, Lim EM, Walsh JP. Associations between body mass index, lean and fat body mass and bone mineral density in middle-aged Australians: the Busselton healthy ageing study. Bone. 2015;74:146-52.

12. Langsetmo L, Hanley DA, Prior JC, Barr SI, Anastassiades T, Towheed T, Goltzman D, Morin S, Poliquin S, Kreiger N. Dietary patterns and incident low-trauma fractures in postmenopausal women and men aged $\geq 50 \mathrm{y}$ : a population-based cohort study. Am J Clin Nutr. 2011;93(1):192-9.

13. Akhlaque U, Ayaz SB, Akhtar N, Ahmad N. Association of bone mineral density and body mass index in a cohort of Pakistanis: relation to gender menopause and ethnicity. The Egyptian Rheumatologist. 2017;39(1):39-43.

14. Butt MS, Sultan MT. Coffee and its consumption: benefits and risks. Crit Rev Food Sci Nutr. 2011;51(4):363-73.

15. Higdon JV, Frei B. Coffee and health: a review of recent human research. Crit Rev Food Sci Nutr. 2006;46(2):101-23.

16. Lin C-C. Approach of improving coffee industry in Taiwan-promote quality of coffee bean by fermentation. JIMS. 2010;5(1):154-9.

17. Demirbag D, Ozdemir F, Ture M. Effects of coffee consumption and smoking habit on bone mineral density. Rheumatol Int. 2006;26(6):530-5.

18. Choi E-J, Kim K-H, Koh Y-J, Lee J-S, Lee D-R, Park SM. Coffee consumption and bone mineral density in Korean premenopausal women. Korean J Fam Med. 2014;35(1):11-8.

19. Hallström H, Byberg L, Glynn A, Lemming EW, Wolk A, Michaëlsson K. Long-term coffee consumption in relation to fracture risk and bone mineral density in women. Am J Epidemiol. 2013:178(6):898-909.

20. Choi $\mathrm{E}$, Choi $\mathrm{K}-\mathrm{H}$, Park SM, Shin D, Joh H-K, Cho E. The benefit of bone health by drinking coffee among Korean postmenopausal women: a cross-sectional analysis of the fourth \& fifth Korea national health and nutrition examination surveys. PLoS One. 2016;11(1):e0147762.

21. Haque M. Sampling methods in social research. Global Research Methodology. 2010:1-6.

22. Nakada M, Demura S. Effect of past and present lifestyle habits and nutrition on calcaneal quantitative osteo-sono index in pre-and post-menopausal females. Health. 2010;2(2):124-30.
23. Arai $Y$, linuma T, Takayama M, Takayama M, Abe Y, Fukuda R, Ando J, Ohta K, Hanabusa H, Asakura K. The Tokyo oldest old survey on Total health (TOOTH): a longitudinal cohort study of multidimensional components of health and well-being. BMC Geriatr. 2010;10(1):35.

24. Tanabe R, Kawamura Y, Tsugawa N, Haraikawa M, Sogabe N, Okano T, Hosoi $\mathrm{T}$, Goseki-Sone M. Effects of Fok-I polymorphism in vitamin D receptor gene on serum 25-hydroxyvitamin D, bone-specific alkaline phosphatase and calcaneal quantitative ultrasound parameters in young adults. Asia Pac J Clin Nutr. 2015:24(2):329-35.

25. Tsuboi S, Hayakawa T, Kanda H, Fukushima T. The relationship between clustering health-promoting components of lifestyle and bone status among middle-aged women in a general population. Environ Health Prev Med. 2009;14(5):292

26. Sugawara N, Yasui Furukori N, Fujii A, Saito M, Sato Y, Nakagami T, Tsuchimine S, Kaneko S. No association between bone mass and prolactin levels among patients with schizophrenia. Hum Psychopharm Clin. 2011;26(8):596-601.

27. Orwoll ES, Bliziotes M. Osteoporosis: pathophysiology and clinical management: Springer Science \& Business Media; 2002.

28. Chin K-Y, Low NY, Dewiputri WI, Ima-Nirwanaa S. Factors associated with bone health in Malaysian middle-aged and elderly women assessed via quantitative ultrasound. Int J Environ Res Public Health. 2017;14(7):736.

29. Chang $\mathrm{H}-\mathrm{C}$, Hsieh $\mathrm{C}-\mathrm{F}$, Lin $\mathrm{Y}-\mathrm{C}$, Tantoh DM, Kung $\mathrm{Y}-\mathrm{Y}$, Lin $\mathrm{M}-\mathrm{C}$, Liaw Y-C, Liaw Y-P. Coffee consumption might reduce the risk of osteopenia/osteoporosis in premenopausal Taiwanese women. JFNR. 2017;5(10):789-93.

30. Medraś M, Jankowska E, Rogucka E. The effect of smoking tobacco and drinking of alcohol and coffee on bone mineral density of healthy men 40 years of age. Pol Arch Med Wewn. 2000;103(3-4):187-93.

31. Hallström H, Melhus H, Glynn A, Lind L, Syvänen A-C, Michaëlsson K. Coffee consumption and CYP1A2 genotype in relation to bone mineral density of the proximal femur in elderly men and women: a cohort study. Nutr Metab. 2010;7(1):12

32. Kapetanovic A, Avdic D. Influence of coffee consumption on bone mineral density in postmenopausal women with estrogen deficiency in menstrual history. J Health Sci. 2014:4:2

33. Yang $P$, Zhang $X-Z$, Zhang $K$, Tang Z. Associations between frequency of coffee consumption and osteoporosis in Chinese postmenopausal women. Int J Clin Exp Med. 2015:8(9):15958.

34. Tang QY, Kukita T, Ushijima Y, Kukita A, Nagata K, Sandra F, Watanabe T, Toh K, Okuma Y, Kawasaki S. Regulation of osteoclastogenesis by Simon extracts composed of caffeic acid and related compounds: successful suppression of bone destruction accompanied with adjuvant-induced arthritis in rats. Histochem Cell Biol. 2006:125(3):215-25.

35. Hallström H, Wolk A, Glynn A, Michaëlsson K. Coffee, tea and caffeine consumption in relation to osteoporotic fracture risk in a cohort of Swedish women. Osteoporosis Int. 2006;17(7):1055-64.

36. Hallström H, Wolk A, Glynn A, Michaëlsson K, Byberg L. Coffee consumption and risk of fracture in the cohort of Swedish men (COSM). PLoS One. 2014; 9(5):e97770.

37. Marques EA, Mota J, Carvalho J. Exercise effects on bone mineral density in older adults: a meta-analysis of randomized controlled trials. Age. 2012;34(6): 1493-515.

38. Langsetmo L, Hitchcock C, Kingwell E, Davison K, Berger C, Forsmo S, Zhou W, Kreiger N, Prior J. Physical activity, body mass index and bone mineral density-associations in a prospective population-based cohort of women and men: the Canadian multicentre osteoporosis study (CaMos). Bone. 2012; 50(1):401-8.

39. Khoo C, Woo J, Leung P, Kwok A, Kwok T. Determinants of bone mineral density in older postmenopausal Chinese women. Climacteric. 2011;14(3): 378-83.

40. Prabha $V$, StanlyA M. Effect of body mass index on bone mineral density; 2015

41. Barrera G, Bunout D, Gattás V, de la Maza MP, Leiva L, Hirsch S. A high body mass index protects against femoral neck osteoporosis in healthy elderly subjects. Nutrition. 2004;20(9):769-71.

42. Wong SK, Chin K-Y, Suhaimi FH, Ahmad F, Ima-Nirwana S. The relationship between metabolic syndrome and osteoporosis: a review. Nutrients. 2016; 8(6):347.

43. Fawzy T, Muttappallymyalil J, Sreedharan J, Ahmed A, Alshamsi SOS, Al All MSSHBB, Al Balsooshi KA. Association between body mass index and bone mineral density in patients referred for dual-energy $X$-ray absorptiometry scan in Ajman, UAE. J Osteoporos. 2011;2011. 
44. Pagotto V, Silveira EA: Bone mineral density in the noninstitutionalized elderly: influence of sociodemographic and anthropometric factors. Curr Gerontol Geriatr Res. 2016, 2016.

45. Ho SC, Y-m C, Woo JL. Educational level and osteoporosis risk in postmenopausal Chinese women. Am J Epidemiol. 2005;161(7):680-90.

46. Chin K-Y, Ima-Nirwana S. Calcaneal quantitative ultrasound as a determinant of bone health status: what properties of bone does it reflect? Int J Med Sci. 2013;10(12):1778.

Ready to submit your research? Choose BMC and benefit from:

- fast, convenient online submission

- thorough peer review by experienced researchers in your field

- rapid publication on acceptance

- support for research data, including large and complex data types

- gold Open Access which fosters wider collaboration and increased citations

- maximum visibility for your research: over $100 \mathrm{M}$ website views per year

At BMC, research is always in progress.

Learn more biomedcentral.com/submissions 